RE: MS\#AJG-15-0022

\title{
Serum Wisteria Floribunda Agglutinin-positive Mac-2-binding Protein Level Predicts Liver Fibrosis and Prognosis in Primary Biliary
}

\section{Cirrhosis}

Short Title: Predictive abilities of WFA ${ }^{+}-M 2 B P$ in PBC

Takeji Umemura, $\mathrm{MD}^{1 *}$ Satoru Joshita, $\mathrm{MD}^{1^{*}}$ Tomohiro Sekiguchi, MD ${ }^{1}$ Yoko Usami, $\mathrm{PhD}^{2}$ Soichiro Shibata, MD ${ }^{1}$ Takefumi Kimura, MD $^{1}$ Michiharu Komatsu, MD ${ }^{1}$ Akihiro Matsumoto, $\mathrm{MD}^{1}$ Masao Ota, $\mathrm{PhD}^{3}$ and Eiji Tanaka, $\mathrm{MD}^{1}$

* contributed equally

1: Department of Medicine, Division of Hepatology and Gastroenterology, Shinshu University School of Medicine, Matsumoto, Japan; 2: Department of Laboratory Medicine, Shinshu University School of Medicine, Matsumoto, Japan; 3: Department of Legal Medicine, Shinshu University Hospital, Matsumoto, Japan

Corresponding Author: Takeji Umemura, MD, PhD, Department of Medicine, Shinshu University School of Medicine, 3-1-1 Asahi, Matsumoto 390-8621, Japan

E-mail: tumemura@shinshu-u.ac.jp; Tel: +81-263-37-2634; Fax: +81-263-32-9412

Word Count: 4,437 words 


\section{Study Highlights}

\section{WHAT IS CURRENT KNOWLEDGE}

- Non-invasive biomarkers are needed to better assess liver fibrosis and predict the progression of primary biliary cirrhosis (PBC).

- The Wisteria floribunda agglutinin-positive Mac-2-binding protein (WFA+-M2BP) has recently been established as a liver fibrosis glycobiomarker.

\section{WHAT IS NEW HERE}

- WFA+-M2BP is independently associated with liver fibrosis stage as determined by liver biopsy in PBC.

- WFA+-M2BP is significantly superior to other non-invasive indices for determination of fibrosis stage.

- WFA+-M2BP level at enrollment is independently associated with clinical outcome. 


\section{ABSTRACT}

OBJECTIVES: Non-invasive markers of liver fibrosis in patients with primary biliary cirrhosis $(\mathrm{PBC})$ are needed for predicting disease progression. Since the Wisteria floribunda agglutinin-positive Mac-2-binding protein (WFA+-M2BP) was recently established as a liver fibrosis glycobiomarker in chronic hepatitis C, we assessed its efficacy in evaluating liver fibrosis stage and disease progression in PBC.

METHODS: A total of 137 patients with PBC who underwent liver biopsy and serological tests for WFA+-M2BP were enrolled. All patients were treated with ursodeoxycholic acid. Clinical data were compared with those for other non-invasive markers (aspartate aminotransferase-to-platelet ratio, FIB-4 index, aspartate aminotransferase/alanine aminotransferase ratio, Forn's index, and Mayo score) for estimating liver fibrosis using receiver operating characteristic analysis. The association between WFA+-M2BP and clinical outcome (liver decompensation, liver transplantation, or death) was evaluated using the Cox proportional hazards model with stepwise method.

RESULTS: WFA+-M2BP was independently associated with liver fibrosis stage as determined by liver biopsy. The cutoff values of WFA+-M2BP for fibrosis stages $\geq F 1, \geq F 2$, $\geq F 3$, and F4 were $0.7,1.0,1.4$, and 2.0 , respectively. The area under the receiver operating characteristic curve values for significant fibrosis, severe fibrosis, and cirrhosis were $0.979,0.933$, and 0.965 , respectively. WFA+-M2BP was significantly superior to the other indices for the determination of significant and severe fibrosis stages. Furthermore, WFA ${ }^{+}-M 2 B P$ level at enrollment was strongly and independently associated with clinical outcome (odds ratio $18.59, P=0.021$ ).

CONCLUSION: Baseline measurements of WFA ${ }^{+}-M 2 B P$ represent a simple and reliable 
non-invasive surrogate marker of liver fibrosis and prognosis in patients with PBC.

Keywords: Autoimmune Liver Disease; Glycoprotein; Biomarker 


\section{INTRODUCTION}

Primary biliary cirrhosis (PBC) is an autoimmune liver disease characterized by portal inflammation and immune-mediated destruction of intrahepatic bile ducts that often leads to cirrhosis and liver failure. $(1,2)$ The course of PBC is highly variable, ranging from several years for rapidly progressive cases to a normal life expectancy in a proportion of asymptomatic patients. The diagnosis of PBC is based on established criteria that include a cholestatic serum biochemical liver test, positivity for anti-mitochondrial antibody, and liver biopsy findings.(3) Although liver biopsy is the gold standard to assess the degree of liver fibrosis, it is often limited by invasiveness and pain, sampling error, and inter-observer disparity. $(4,5)$ Biopsy of the liver may be indicated in a small portion of patients in whom diagnostic features are atypical for PBC.(6) As severe fibrosis and cirrhosis are generally related to a poor outcome,(2) simple and reliable non-invasive methods are urgently needed to better assess liver fibrosis and predict the progression of the disease.

Recently, Wisteria floribunda agglutinin-positive Mac-2 binding protein (WFA $\left.{ }^{+}-\mathrm{M} 2 \mathrm{BP}\right)$ was reported as a novel, non-invasive, and rapid bedside method of estimating liver fibrosis.(7) M2BP contains multibranching and sialylated $\mathrm{N}$-glycans, and WFA is considered to recognize the GalNAc residue of N-glycans and O-glycans or the clustered LacNAc (Gal-GlcNAc) structure. Alterations in M2BP during the progression of liver disease and fibrosis are presumed to be due to changes in $N$-glycosylation. Moreover, M2BP binds to galectin-3, $\beta-1$ integrins, collagens, and fibronection and has relevance in cell-cell and cell-extracellular matrix adhesion.(8, 9) It is for these reasons that measurements of serum hyperglycosylated WFA-M2BP by glycan-based 
immunoassays are currently postulated to reflect the progression of fibrosis. The $W_{F A}^{+}-M 2 B P$ method has been validated for chronic hepatitis $C(10)$ and non-alcoholic fatty liver disease,(11) and may also predict the development of hepatocellular carcinoma.(12) However, its ability to reflect extensive fibrosis and prognosis in PBC remains to be demonstrated. The objective of this study was to assess the diagnostic performance of the WFA+-M2BP assay for the evaluation of fibrosis and long-term clinical outcome in PBC.

\section{METHODS}

\section{Subjects}

A total of 303 patients were diagnosed as having PBC between January 1981 and June 2014 at Shinshu University Hospital or its affiliated institutions. Among them, 166 patients were excluded from this retrospective analysis for the following reasons: (1) no liver histology data $(n=84)$; (2) no stored serum from prior to ursodeoxycholic acid (UDCA) administration ( $n=62$ ); (3) other chronic liver disease, such as hepatitis B, hepatitis C, or non-alcoholic steatohepatitis $(n=15)$; and (4) follow-up $<6$ months $(n=5)$. The remaining 137 uniformly Japanese patients were enrolled in the present study. The characteristics of the 137 biopsied patients and 84 non-biopsied patients are summarized in supplementary Table 1. The diagnosis of PBC was based on criteria from the American Association for the Study of Liver Diseases.(3) All patients were treated with UDCA (10-13 mg/kg per day). Forty-five healthy subjects with normal liver function tests served as controls. In addition, 40 Japanese patients with type 1 autoimmune hepatitis $(\mathrm{AlH})$ were adopted as comparison cases having another autoimmune liver 
disease. All patients and controls were negative for hepatitis B surface antigen and antibodies to hepatitis B core antigen, hepatitis C virus, and the human immunodeficiency virus. Patients who exhibited evidence of other liver disease during follow-up, such as alcoholic or non-alcoholic liver disease, were excluded from the cohort. This study was conducted in accordance with the principles of the 1975 Declaration of Helsinki. The protocol was reviewed and approved by the institutional ethics committee. Serum samples were obtained at the time of liver biopsy prior to UDCA treatment and stored at $-30^{\circ} \mathrm{C}$ until testing.

\section{Laboratory Testing}

All laboratory data were obtained on the same day as the liver biopsy. Alanine aminotransferase (ALT), aspartate aminotransferase (AST), alkaline phosphatase, Y-glutamyltransferase, and other relevant biochemical tests were performed using standard methods. Four additional surrogate blood indices of liver fibrosis were assessed at enrollment: AST-to-platelet ratio (APRI), FIB-4 index, AST/ALT ratio, and Forn's index. APRI,(13) FIB-4 index,(14) and Forn's index(15) were calculated according to published formulas. The Mayo clinic risk score for PBC was also tested as a potential surrogate marker of liver fibrosis.(16) Anti-nuclear antibody titer was determined by immunofluorescence using HEp-2 cells, whereby a titer of 1:80 or greater was considered as positive.(17) Serum anti-mitochondrial antibody, which is specific for the pyruvate dehydrogenase complex-E2 component, was measured using an enzyme-linked immunosorbent assay. An index of greater than 7 was considered to be a positive result.(18) 


\section{Liver Biopsy and Histological Evaluation}

Liver biopsies were performed by percutaneous sampling of the right lobe with a 14-gauge needle in all patients but 7 before administration of UDCA. All biopsies were $1.5 \mathrm{~cm}$ or more in length. Formalin-fixed and paraffin-embedded specimens were prepared and used for histopathological studies. Sections measuring $4 \mu \mathrm{m}$ were cut from each paraffin block and stained with hematoxylin and eosin, periodic acid-Schiff after diastase digestion, and Azan-Mallory staining. For the 7 patients who did not undergo liver biopsy, nodular liver laparoscopic findings had already indicated definite cirrhosis. Histological stage was determined according to Scheuer's(19) classification. Liver fibrosis and necroinflammatory activity were judged using the METAVIR scoring system,(20) wherein significant fibrosis was defined as $\geq F 2$, severe fibrosis as $\geq F 3$, and cirrhosis as F4. The degree of bile duct loss ( 0 , absent; $1,1 / 3$ of portal tract; $2,2 / 3$ of portal tract; and $3,>2 / 3$ of portal tract) was evaluated as described by Nakanuma et al.(21) The investigators (AM, ET) involved in this part of the study were blinded to the results of other portions.

\section{Measurement of WFA+-M2BP}

WFA+-M2BP was quantified based on a lectin-antibody sandwich immunoassay using a fully automatic HISCL-5000 immunoanalyzer (Sysmex Co., Hyogo, Japan).(7) The measured values of WFA+-M2BP conjugated to WFA were indexed with obtained values using the following equation: Cutoff index $=\left(\left[\mathrm{WFA} A^{+}-M 2 B P\right]_{\text {sample }}-\right.$ $\left.\left[\mathrm{WFA}^{+}-\mathrm{M} 2 \mathrm{BP}\right]_{\mathrm{NC}}\right) /\left(\left[\mathrm{WFA}{ }^{+}-\mathrm{M} 2 \mathrm{BP}\right]_{\mathrm{PC}}-\left[\mathrm{WFA}^{+}-\mathrm{M} 2 \mathrm{BP}\right]_{\mathrm{NC}}\right)$ where $\left[\mathrm{WFA}^{+}-\mathrm{M} 2 \mathrm{BP}\right]_{\text {sample }}$ was 
the WFA $\mathrm{W}^{+}-\mathrm{M} 2 \mathrm{BP}$ count of the serum sample, $\mathrm{PC}$ was positive control, and $\mathrm{NC}$ was negative control. The positive control was supplied as a calibration solution preliminarily standardized to yield a cutoff index value of 1.0.(22)

\section{Statistical Analysis}

Categorical variables were compared using the chi-squared or Fisher's exact test, as appropriate, and continuous variables were compared with the Mann-Whitney $U$ test. Spearman's rank order correlations were used to evaluate serum WFA+-M2BP levels with clinical features. Stepwise multiple linear regression analysis was performed to identify the independent factors influencing serum WFA+-M2BP level. The diagnostic performance of WFA ${ }^{+}-\mathrm{M} 2 \mathrm{BP}$ was determined in terms of sensitivity, specificity, positive and negative predictive values, and area under the receiver operating characteristic curve (AUC). Optimal cutoff values were determined by the Youden index. Comparison of paired AUCs and 95\% confidence intervals was carried out using the nonparametric Delong test.(23)

Clinical outcome as of June 2014 was recorded as either liver-related death, liver transplantation, or liver decompensation, such as ascites, esophageal varices, hepatic encephalopathy, hepatocellular carcinoma, or total serum bilirubin level above $3 \mathrm{mg} / \mathrm{dL}$. The Kaplan-Meier method and log-rank test were used to estimate the survival rate of patients. Multivariate analysis was performed using the Cox proportional hazards model with stepwise method. A $P<0.05$ was considered to be significant. Statistical analyses were performed using IBM SPSS Statistics 21.0 (IBM, Chicago, IL) and StatFlex ver. 6.0 (Artech Co., Ltd. Osaka, Japan) software. 


\section{RESULTS}

\section{Baseline Clinical Characteristics of Patients}

The clinical profile of the experimental patient cohort is shown in Table 1. Median age was 57 years with a female predominance (84\%). Median follow-up was 7.7 years (interquartile range [IQR], 3.7-13.8 years). The median (IQR) values for the FIB-4 index, APRI, AST/ALT ratio, Forn's index, and Mayo risk score were 1.6 (1.2-2.3), 4.7 (3.1-6.5), 1.0 (0.8-1.2), 5.7 (4.8-6.9), and $4.2(3.7-4.6)$, respectively. Fibrosis stage was F0 for 10 cases (7\%), F1 for 71 cases (52\%), F2 for 27 cases (20\%), F3 for 18 cases (13\%), and F4 for 11 cases $(8 \%)$.

\section{Correlation between WFA+-M2BP and Liver Fibrosis in Patients with PBC}

Serum samples from 137 patients with PBC at diagnosis, 45 healthy subjects, and 40 patients with type $1 \mathrm{AlH}$ were examined for levels of WFA+-M2BP. Median serum WFA+-M2BP was significantly higher in PBC patients $(0.8 ; I Q R, 0.5-1.5)$ than in controls $(0.2 ; \mathrm{IQR}, 0.1-0.3)(P<0.001)$ but similar to that in AlH subjects $(1.1 ; \mathrm{IQR}, 0.8-1.8)$. Figure 1 presents box plots of WFA+-M2BP levels according to each fibrosis stage. Among the PBC patients, median serum WFA+-M2BP increased in association with progression of fibrosis score as 0.4 (F0), 0.6 (F1), 1.4 (F2), 2.0 (F3), and 3.0 (F4). There was a significant association between WFA+-M2BP and fibrosis stage in PBC (rho = 0.841; $P<0.001$ ) (Table 2), which persisted when WFA ${ }^{+}-M 2 B P$ levels in healthy subjects were added to the analysis (rho $=0.903 ; P<0.001$ ). Moreover, significant differences were noted for WFA+-M2BP when each fibrosis stage was compared (Supplementary 
Table 2).

\section{Correlation between WFA+-M2BP and Clinical Features}

Demographic (age, gender), clinical (platelet count, total protein, albumin, ALT, AST, alkaline phosphatase, $\mathrm{y}$-glutamyltransferase, bilirubin, $\lg \mathrm{M}, \lg \mathrm{G}$, anti-mitochondrial antibody, anti-nuclear antibody, FIB-4 index, APRI, AST/ALT ratio, Forn's index, Mayo score), and histological (fibrosis stage, activity grade, loss of bile duct, Scheuer's stage) findings were examined for their correlation with WFA+-M2BP level in patients with PBC (Table 2). Serum WFA+-M2BP was significantly correlated with ALT, AST, alkaline phosphatase, y-glutamyltransferase, bilirubin, IgM, IgG, FIB-4 index, APRI, Forn's index, Mayo score, and all histological results and was inversely correlated with serum albumin.

\section{Diagnostic Performance of WFA+-M2BP}

Receiver operating characteristic curve analysis was performed to determine the diagnostic accuracy of WFA+-M2BP for each stage of fibrosis in PBC. The calculated AUC, optimal cutoff value, sensitivity, specificity, positive predictive value, and negative predictive value for each fibrosis stage are listed in Table 3. AUCs were 0.883, 0.979, 0.933 , and 0.965 for $\geq F 1, \geq F 2, \geq F 3$, and $F 4$, respectively. The optimal cutoff values that best predicted fibrosis stages $\geq F 1, \geq F 2$, $\geq F 3$, and $F 4$ were $0.7,1.0,1.4$, and 2.0, respectively. Overall, WFA ${ }^{+}-\mathrm{M} 2 \mathrm{BP}$ was found to be of high reliability for the diagnosis of significant fibrosis, severe fibrosis, and cirrhosis.

We next performed univariate and multivariate analyses of factors associated 
with the presence of significant fibrosis $(\geq F 2)$ (Supplementary Table 3 ). The variables of total protein, ALT, AST, alkaline phosphatase, y-glutamyltransferase, bilirubin, IgM, IgG, anti-mitochondrial antibody positivity, WFA+-M2BP $>1.0$, FIB-4 index $>1.8$, APRI $>0.5$, AST/ALT ratio $>1.1$, and Forn's index $>6.9$ were significantly higher in the significant fibrosis group than in the non-significant fibrosis group, whereas platelet count and albumin were significantly lower in the significant fibrosis group. Among these, IgG, anti-mitochondrial antibody positivity, WFA ${ }^{+}-M 2 B P$, and Forn's index were independent variables associated with significant fibrosis according to multivariate logistic regression analysis.

\section{Comparison of Fibrosis Marker AUCs}

The receiver operating characteristic curves for WFA+-M2BP, FIB-4 index, APRI, AST/ALT ratio, Forn's index, and Mayo score for predicting significant fibrosis $(\geq F 2)$, severe fibrosis $(\geq \mathrm{F} 3)$, and cirrhosis (F4) are shown in Figures $2 \mathrm{~A}-\mathrm{C}$. The AUC of WFA $^{+}-\mathrm{M} 2 \mathrm{BP}$ for predicting significant fibrosis $(0.979)$ was significantly superior to those of FIB-4 index $(0.720, P<0.001)$, APRI $(0.754, P<0.001)$, AST/ALT ratio $(0.613, P<$ $0.001)$, Forn's index $(0.640, P<0.001)$, and Mayo score $(0.716, P<0.001)$. Similarly, the AUC of WFA+-M2BP (0.933) was superior to those of FIB-4 index $(0.713, P<0.001)$, APRI $(0.837, P=0.032)$, AST/ALT ratio $(0.589, P<0.001)$, Forn's index $(0.609, P<$ $0.001)$, and Mayo score $(0.733, P<0.001)$ for the prediction of severe fibrosis. For estimating cirrhosis, the AUC of WFA+-M2BP (0.965) was significantly superior to those of FIB-4 index $(0.829, P=0.027)$, APRI $(0.820, P=0.003)$, AST/ALT ratio $(0.823, P=$ 0.05), and Forn's index $(0.724, P=0.040)$ and comparable to that of Mayo score $(0.960)$. 
Prediction and Risk Factors of Liver-related Death, Transplantation, and Liver Decompensation at Diagnosis

The results of multivariate Cox regression analysis regarding factors associated with liver-related death or liver transplantation are shown in Table 4 and reveal WFA $^{+}-\mathrm{M} 2 \mathrm{BP}$ to be a significant predictor of these outcomes (odds ratio $18.59 ; 95 \% \mathrm{Cl}$ : 1.57-220.51, $P=0.021$ ). Moreover, in Kaplan-Meier estimates of liver-related death or transplantation at different WFA+-M2BP levels at diagnosis, the 10-year survival rate was $98.8 \%$ in patients with a low-risk WFA+-M2BP level $(<2.0)$, versus $59.1 \%$ in those with a high-risk WFA+-M2BP value ( $\geq 2.0)$ (Fig. 3). The cumulative patient survival rate between these two groups was significantly different (log-rank test; $P<0.001)$.

Multivariate Cox regression analysis regarding factors associated with liver decompensation is summarized in Table 5 and shows WFA ${ }^{+}-M 2 B P$ to be a significant predictor of this outcome (odds ratio $18.59 ; 95 \% \mathrm{Cl}: 1.57-220.51, P=0.021$ ). In Kaplan-Meier estimates of liver decompensation at different WFA+-M2BP levels at diagnosis, the cumulative liver decompensation rate between the low-risk and high-risk groups was significantly different (log-rank test; $P<0.001)$ (Fig. 3B). Mayo risk score was not remarkably associated with death or decompensation in univariate and multivariate analyses.

\section{DISCUSSION}

This study clearly demonstrated that WFA+-M2BP level in PBC was significantly higher than that in healthy controls and increased with progression of fibrosis stage, 
which was in agreement with several studies regarding WFA+-M2BP and chronic hepatitis C. $(7,10,12)$ As a liver fibrosis glycobiomarker with a fully automated immunoassay, WFA+-M2BP showed high specificity and sensitivity in predicting significant and severe fibrosis and cirrhosis in PBC. In addition, stepwise multiple regression analysis revealed that liver fibrosis stage was most closely associated with serum WFA+-M2BP level. Histological liver data are generally not available for PBC due to the current standard of care minimizing the use of liver biopsy. Although our sample number, and especially the proportion of severe fibrosis $(\geq F 3)$ and cirrhosis (F4) subjects, was relatively small, WFA+-M2BP appears to be a highly predictive fibrosis marker.

Our data confirmed that the WFA+-M2BP assay was strongly associated with other non-invasive markers of fibrosis, as well with liver function tests. Furthermore, the assay was significantly superior to the above fibrosis markers for identifying significant $(\geq F 2)$ and severe $(\geq F 3)$ fibrosis. We witnessed two key features about the WFA+-M2BP assay that were distinct from the other published fibrosis markers. First, the AUC of the assay was as high as 0.98 for the F2 stage. In contrast, the AUCs of previously reported fibrosis markers gradually increased as fibrosis progressed. This suggests that the WFA ${ }^{+}-M 2 B P$ assay may be a particularly good marker to differentiate early from significant fibrosis (Supplementary Table 2). Second, the range of the WFA+-M2BP serum thresholds observed in PBC patients was different from those described in chronic hepatitis $C(7,10,12)$ but were similar to those in non-alcoholic fatty liver disease (11), $\mathrm{AlH}$, and hepatitis B (Matsumoto and Umemura, personal communications). The reasons for lower WFA+-M2BP levels in PBC are unknown, however, individual cut-off values for each fibrosis stage should be determined for all liver diseases. 
Although several fibrosis markers have been assessed for PBC, $(24,25)$ none have been established as surrogate markers in lieu of biopsy; only the European liver fibrosis test, which includes serum hyaluronic acid, tissue inhibitor of metalloproteinase 1 , and $\mathrm{N}$-terminal propeptide of type III procollagen, has shown acceptable diagnostic accuracy for significant fibrosis or cirrhosis.(26) Since the European liver fibrosis test was not performed in our cohort, a comparison of this marker with WFA+-M2BP is needed in the future. Transient elastography has also been described as one of the best non-invasive markers of liver fibrosis in PBC.(27-29) Whereas the diagnostic accuracy of this technique is high,(29) $10-20 \%$ of transient elastography results are unreliable when the patient is obese or exhibits ascites. $(29,30)$ Operator skill affects its diagnostic success rate as well, indicating that there remain some limitations for transient elastography. As WFA+-M2BP combined with transient elastography may provide the highest accuracy for fibrosis assessment in $\mathrm{PBC}$, we are now conducting prospective studies on dual testing.

Another important conclusion from this study is that WFA+-M2BP at diagnosis may be an independent prognostic factor for PBC. Several variables have been proposed to contribute to PBC progression, such as anti-gp210 antibody(31) and HLA haplotype(32) in the Japanese. The Mayo risk score appeared to be a good surrogate marker for predicting poor outcome in patients possessing symptomatic PBC with advanced fibrosis, but risk score at diagnosis was not associated with adverse outcome in our cohort, possibly because of the large proportion of asymptomatic PBC and mild fibrosis. Very recently, a collaborative international multicenter study showed levels of alkaline phosphatase and bilirubin at diagnosis to be prognostic markers for PBC in 
North American and European countries.(33) However, these parameters were not identified as significant factors related to an unfavorable outcome in the present investigation. This could be due to the small number of poor outcomes in this study or because it was underpowered to assess prognosis. Another explanation may be the different ethnicities among study populations or the fact that the majority of our cohort was asymptomatic and exhibited mild fibrosis.

Lastly, we noticed that a high-risk WFA+-M2BP level $(\geq 2.0)$ was associated with elevated long-term mortality in PBC. The identification of PBC with a poor long-term outcome among patients treated with adequate doses of UDCA is an important issue in clinical practice as well as in the design of therapeutic trials; some patients with an incomplete response to UDCA develop a progressive disease condition that eventually leads to liver transplantation or death from liver-related causes. A number of prognostic models following UDCA treatment have been shown to serve as independent predictors of transplant-free and overall survival.(33-39) Zhang et al.(40) demonstrated that the biochemical responses at 6 months could represent those at 1 year after UDCA therapy. Since our aim was to estimate the prognosis of PBC at enrollment, the biochemical response to UDCA was not included in this investigation. Another report has shown that advanced histological stage determined by transient elastography at enrollment is also a strong prognostic indicator of PBC.(29) Our data suggest that evaluating WFA - M2BP at entry is an easy and useful tool not only for predicting fibrosis stage in PBC, but also for estimating prognosis.

The limitations of this study are its retrospective nature and lack of a validation cohort despite a long median follow-up period of 7.7 years. In addition, only $9(7 \%)$ of our 
137 patients experienced liver-related death or transplantation. Since repeated liver biopsies during follow-up were not conducted, the actual correlations between the changes in WFA+-M2BP and fibrosis could not be determined. Future prospective trials in larger cohorts that include various ethnicities are required for the WFA+-M2BP assay.

In conclusion, this study demonstrated the accuracy and usefulness of WFA $^{+}-\mathrm{M} 2 \mathrm{BP}$ for the assessment of liver fibrosis in patients with PBC, which was consistent with recent results obtained for chronic hepatitis C. WFA+-M2BP appears to be a simple, reliable, and non-invasive method for evaluating fibrosis and predicting clinical outcome in PBC.

\section{ACKNOWLEDGMENTS}

The authors thank Trevor Ralph for his English editorial assistance. The Sysmex Co. provided all materials for detecting WFA+-M2BP.

\section{CONFLICT OF INTEREST/STUDY SUPPORT}

Guarantor of the Article: Takeji Umemura, MD

Specific Author Contributions: Study concept and design (Takeji Umemura, Satoru Joshita) ; acquisition of data (Takeji Umemura, Satoru Joshita, Tomohiro Sekiguchi, Yoko Usami, Soichiro Shibata, Takefumi Kimura, Michiharu Komatsu, Akihiro Matsumoto); analysis and interpretation of data (Takeji Umemura, Satoru Joshita, Masao Ota, Eiji Tanaka); drafting of the manuscript (Takeji Umemura); critical revision of the manuscript for important intellectual content (Satoru Joshita, Masao Ota, Eiji Tanaka); statistical analysis (Satoru Joshita). All authors have approved the final version of the article.

Financial Support: This work was supported in part by Grants-in-Aid for Scientific Research from the Ministry of Education, Science, Sports, and Culture of Japan 
(26460996).

Potential Competing Interests: Eiji Tanaka received a research grant from Sysmex Co. 


\section{References}

1. Kaplan MM, Gershwin ME. Primary biliary cirrhosis. N Engl J Med 2005;353:1261-73.

2. Selmi C, Bowlus CL, Gershwin ME, et al. Primary biliary cirrhosis. Lancet 2011;377:1600-9.

3. Lindor KD, Gershwin ME, Poupon R, et al. Primary biliary cirrhosis. Hepatology 2009;50:291-308.

4. $\quad$ Cadranel JF, Rufat P, Degos F. Practices of liver biopsy in France: results of a prospective nationwide survey. For the Group of Epidemiology of the French Association for the Study of the Liver (AFEF). Hepatology 2000;32:477-81.

5. Bravo AA, Sheth SG, Chopra S. Liver biopsy. N Engl J Med 2001;344:495-500.

6. Zein CO, Angulo P, Lindor KD. When is liver biopsy needed in the diagnosis of primary biliary cirrhosis? Clin Gastroenterol Hepatol 2003;1:89-95.

7. Kuno A, Ikehara Y, Tanaka Y, et al. A serum "sweet-doughnut" protein facilitates fibrosis evaluation and therapy assessment in patients with viral hepatitis. Sci Rep 2013;3:1065.

8. Sasaki T, Brakebusch C, Engel J, et al. Mac-2 binding protein is a cell-adhesive protein of the extracellular matrix which self-assembles into ring-like structures and binds beta1 integrins, collagens and fibronectin. EMBO J 1998;17:1606-13.

9. Inohara $\mathrm{H}$, Akahani S, Koths $\mathrm{K}$, et al. Interactions between galectin-3 and Mac-2-binding protein mediate cell-cell adhesion. Cancer Res 1996;56:4530-4.

10. Toshima T, Shirabe K, Ikegami T, et al. A novel serum marker, glycosylated Wisteria floribunda agglutinin-positive Mac-2 binding protein (WFA-M2BP), for assessing liver fibrosis. J Gastroenterol 2014.

11. Abe M, Miyake T, Kuno A, et al. Association between Wisteria floribunda agglutinin-positive Mac-2 binding protein and the fibrosis stage of non-alcoholic fatty liver disease. J Gastroenterol 2014.

12. Yamasaki K, Tateyama M, Abiru S, et al. Elevated serum levels of WFA -M2BP predict the development of hepatocellular carcinoma in hepatitis C patients. Hepatology 2014.

13. Wai CT, Greenson JK, Fontana RJ, et al. A simple noninvasive index can predict both significant fibrosis and cirrhosis in patients with chronic hepatitis C. Hepatology 2003;38:518-26.

14. Sterling RK, Lissen E, Clumeck N, et al. Development of a simple noninvasive index to predict significant fibrosis in patients with HIV/HCV coinfection. Hepatology 2006;43:1317-25.

15. Forns X, Ampurdanes S, Llovet JM, et al. Identification of chronic hepatitis C patients without hepatic fibrosis by a simple predictive model. Hepatology 2002;36:986-92.

16. Dickson ER, Grambsch PM, Fleming TR, et al. Prognosis in primary biliary cirrhosis: model for decision making. Hepatology 1989;10:1-7.

17. Umemura T, Katsuyama Y, Yoshizawa K, et al. Human leukocyte antigen class II haplotypes affect clinical characteristics and progression of type 1 autoimmune hepatitis in Japan. PLoS One 2014;9:e100565.

18. Umemura T, Zen Y, Hamano H, et al. Immunoglobin G4-hepatopathy: association of immunoglobin G4-bearing plasma cells in liver with autoimmune pancreatitis. Hepatology 2007;46:463-71.

19. Scheuer P. Primary biliary cirrhosis. Proc R Soc Med 1967;60:1257-60.

20. Bedossa P, Poynard T. An algorithm for the grading of activity in chronic hepatitis $C$. The METAVIR Cooperative Study Group. Hepatology 1996;24:289-93.

21. Hiramatsu K, Aoyama H, Zen $\mathrm{Y}$, et al. Proposal of a new staging and grading system of the liver for primary biliary cirrhosis. Histopathology 2006;49:466-78.

22. Kuno A, Sato T, Shimazaki H, et al. Reconstruction of a robust glycodiagnostic agent supported by multiple lectin-assisted glycan profiling. Proteomics Clin Appl 2013.

23. DeLong ER, DeLong DM, Clarke-Pearson DL. Comparing the areas under two or more correlated receiver operating characteristic curves: a nonparametric approach. Biometrics 1988;44:837-45.

24. Farkkila M, Rautiainen $\mathrm{H}$, Karkkainen $\mathrm{P}$, et al. Serological markers for monitoring disease progression in noncirrhotic primary biliary cirrhosis on ursodeoxycholic acid therapy. Liver Int 2008;28:787-97.

25. Alempijevic T, Krstic M, Jesic R, et al. Biochemical markers for non-invasive assessment of disease stage in patients with primary biliary cirrhosis. World J Gastroenterol 2009;15:591-4.

26. Mayo MJ, Parkes J, Adams-Huet B, et al. Prediction of clinical outcomes in primary biliary cirrhosis by serum enhanced liver fibrosis assay. Hepatology 2008;48:1549-57.

27. Corpechot C, El Naggar A, Poujol-Robert A, et al. Assessment of biliary fibrosis by transient 
elastography in patients with PBC and PSC. Hepatology 2006;43:1118-24.

28. Gomez-Dominguez E, Mendoza J, Garcia-Buey L, et al. Transient elastography to assess hepatic fibrosis in primary biliary cirrhosis. Aliment Pharmacol Ther 2008;27:441-7.

29. Corpechot C, Carrat F, Poujol-Robert A, et al. Noninvasive elastography-based assessment of liver fibrosis progression and prognosis in primary biliary cirrhosis. Hepatology 2012;56:198-208.

30. Castera L, Foucher J, Bernard PH, et al. Pitfalls of liver stiffness measurement: a 5-year prospective study of 13,369 examinations. Hepatology 2010;51:828-35.

31. Nakamura M, Kondo H, Mori T, et al. Anti-gp210 and anti-centromere antibodies are different risk factors for the progression of primary biliary cirrhosis. Hepatology 2007;45:118-27.

32. Umemura T, Joshita S, Ichijo T, et al. Human leukocyte antigen class II molecules confer both susceptibility and progression in Japanese patients with primary biliary cirrhosis. Hepatology 2012;55:506-11.

33. Lammers WJ, van Buuren HR, Hirschfield GM, et al. Levels of Alkaline Phosphatase and Bilirubin are Surrogate Endpoints of Outcomes of Patients with Primary Biliary Cirrhosis - an International Follow-up Study. Gastroenterology 2014.

34. Pares A, Caballeria L, Rodes J. Excellent long-term survival in patients with primary biliary cirrhosis and biochemical response to ursodeoxycholic Acid. Gastroenterology 2006;130:715-20.

35. Corpechot C, Abenavoli L, Rabahi N, et al. Biochemical response to ursodeoxycholic acid and long-term prognosis in primary biliary cirrhosis. Hepatology 2008;48:871-7.

36. Kuiper EM, Hansen BE, de Vries RA, et al. Improved prognosis of patients with primary biliary cirrhosis that have a biochemical response to ursodeoxycholic acid. Gastroenterology 2009;136:1281-7. 37. Kumagi T, Guindi M, Fischer SE, et al. Baseline Ductopenia and Treatment Response Predict Long-Term Histological Progression in Primary Biliary Cirrhosis. Am J Gastroenterol 2010.

38. Corpechot $\mathrm{C}$, Chazouilleres $\mathrm{O}$, Poupon R. Early primary biliary cirrhosis: biochemical response to treatment and prediction of long-term outcome. J Hepatol 2011;55:1361-7.

39. Trivedi PJ, Bruns T, Cheung A, et al. Optimising risk stratification in primary biliary cirrhosis: AST/platelet ratio index predicts outcome independent of ursodeoxycholic acid response. J Hepatol 2014;60:1249-58.

40. Zhang LN, Shi TY, Shi XH, et al. Early biochemical response to ursodeoxycholic acid and long-term prognosis of primary biliary cirrhosis: results of a 14-year cohort study. Hepatology 2013;58:264-72. 
Table 1. Demographic, Clinical, Biochemical, and Histological Characteristics of 137 Patients with PBC

\begin{tabular}{|c|c|c|}
\hline \multicolumn{3}{|l|}{ Characteristic } \\
\hline Age at entry (years) & 57 & $51-63$ \\
\hline Female, n (\%) & $111(81)$ & \\
\hline Follow-up (years) & 7.7 & $3.7-13.8$ \\
\hline \multicolumn{3}{|l|}{ Laboratory data at entry } \\
\hline Platelet count $\left(\times 10^{9} / \mathrm{L}\right)$ & 223 & $181-256$ \\
\hline Total protein $(6.8-8.3 \mathrm{~g} / \mathrm{dL})$ & 7.7 & $7.4-8.2$ \\
\hline Albumin $(4.2-5.1 \mathrm{~g} / \mathrm{dL})$ & 4.2 & $4.0-4.5$ \\
\hline ALT (7-45 IU/L) & 42 & $28-69$ \\
\hline AST (12-37 IU/L) & 41 & $30-63$ \\
\hline ALP (124-367 IU/L) & 451 & $326-605$ \\
\hline GGT (8-50 IU/L) & 136 & $86-261$ \\
\hline Bilirubin (0.3-1.2 mg/dL) & 0.8 & $0.6-1.0$ \\
\hline $\operatorname{lgM}(35-220$ mg/dL) & 292 & $185-511$ \\
\hline IgG (870-1700 mg/dL) & 1630 & $1359-2005$ \\
\hline AMA positive, n (\%) & $117(85)$ & \\
\hline ANA positive, n (\%) & $97(71)$ & \\
\hline \multicolumn{3}{|l|}{ Fibrosis markers } \\
\hline WFA+-M2BP & 0.8 & $0.5-1.5$ \\
\hline FIB-4 index & 1.6 & $1.2-2.3$ \\
\hline APRI & 4.7 & $3.1-6.5$ \\
\hline AST/ALT ratio & 1.0 & $0.8-1.2$ \\
\hline Forn's index & 5.7 & $4.8-6.9$ \\
\hline Mayo score & 4.2 & $3.7-4.6$ \\
\hline \multicolumn{3}{|l|}{ Histological findings } \\
\hline Fibrosis stage (F0:F1:F2:F3:F4) & \multicolumn{2}{|c|}{$10(7): 71(52): 27(20): 18(13): 11(8)$} \\
\hline Activity grade (A0:A1:A2:A3) & \multicolumn{2}{|c|}{$42(32): 58(45): 27(21): 3(2)$} \\
\hline Loss of bile duct $(0: 1: 2: 3)$ & \multicolumn{2}{|c|}{$78(60): 30(23): 19(15): 3(2)$} \\
\hline Scheuer's stage (I:II:III:IV) & \multicolumn{2}{|c|}{$87(64): 23(17): 16(12): 11(8)$} \\
\hline
\end{tabular}

Parameters are presented as median (IQR) for continuous variables and total number (\%) for categorical variables. Units and normal ranges are stated in parentheses. AMA, anti-mitochondrial antibody; ANA, anti-nuclear antibody; ALP, alkaline phosphatase; ALT, alanine aminotransferase AST, aspartate aminotransferase; GGT, Y-glutamyltransferase. 
Table 2. Correlation between Serum WFA+-M2BP Level and Demographic and Clinical Characteristics in 137 Patients with PBC

\begin{tabular}{lcr}
\hline Characteristic & rho & $\boldsymbol{P}$ value \\
\hline Age & 0.085 & 0.324 \\
Gender & -0.084 & 0.330 \\
Laboratory findings & & \\
Platelet count & -0.092 & 0.284 \\
Total protein & 0.158 & 0.066 \\
Albumin & -0.372 & $<0.001$ \\
ALT & 0.264 & 0.002 \\
AST & 0.383 & $<0.001$ \\
ALP & 0.266 & 0.002 \\
GGT & 0.279 & 0.001 \\
Bilirubin & 0.230 & 0.007 \\
IgM & 0.268 & 0.002 \\
IgG & 0.274 & 0.001 \\
AMA positive & 0.100 & 0.245 \\
ANA positive & 0.000 & 0.998 \\
Fibrosis markers & & \\
FIB-4 index & 0.311 & $<0.001$ \\
APRI & 0.439 & $<0.001$ \\
AST/ALT ratio & 0.157 & 0.068 \\
Forn's index & 0.228 & 0.008 \\
Mayo score & 0.366 & $<0.001$ \\
Histological findings & & \\
Fibrosis stage & 0.841 & $<0.001$ \\
Activity grade & 0.429 & $<0.001$ \\
Loss of bile duct & 0.561 & $<0.001$ \\
Scheuer's stage & 0.503 & $<0.001$ \\
\hline
\end{tabular}


Table 3. Diagnostic Performance of WFA+-M2BP in 137 Patients with PBC

\begin{tabular}{|c|c|c|c|c|c|c|}
\hline Fibrosis stage & Cutoff & AUC (95\% Cl) & Sensitivity (\%) & Specificity (\%) & PPV (\%) & NPV (\%) \\
\hline$\geq F 1$ & 0.7 & $0.883(0.809-0.958)$ & 70 & 100 & 100 & 21 \\
\hline$\geq F 2$ & 1.0 & $0.979(0.956-1.000)$ & 93 & 93 & 90 & 95 \\
\hline$\geq F 3$ & 1.4 & $0.933(0.880-0.986)$ & 83 & 90 & 69 & 95 \\
\hline =F4 & 2.0 & $0.965(0.936-0.994)$ & 39 & 100 & 100 & 70 \\
\hline
\end{tabular}

Cutoff values are determined as the maximum total sensitivity and specificity.

AUC, area under the receiver operating characteristic curve; PPV, positive predictive value; NPV, negative predictive value. 
Table 4. Factors Associated with Liver-related Death or Transplantation in Patients with PBC as Identified by Multivariate Analysis

\begin{tabular}{lccc}
\hline Factor & Category & Odds ratio (95\% Cl) & $P$ value \\
\hline Albumin $(\mathrm{g} / \mathrm{dL})$ & $<4.0$ & 1 & \\
& $\geq 4.0$ & $0.04(0.00-0.41)$ & 0.007 \\
GGT (IU/L) & $<192$ & 1 & \\
& $\geq 192$ & $30.32(1.75-524.46)$ & 0.019 \\
WFA $^{+}-\mathrm{M} 2 \mathrm{BP}$ & $<2.0$ & 1 & 0.021 \\
& $\geq 2.0$ & $18.59(1.57-220.51)$ & \\
\hline
\end{tabular}


Table 5. Factors Associated with Liver Decompensation in Patients with PBC as Identified by Multivariate Analysis

\begin{tabular}{lccc}
\hline Factor & Category & Odds ratio (95\% Cl) & $P$ value \\
\hline Albumin $(\mathrm{g} / \mathrm{dL})$ & $<4.0$ & 1 & \\
& $\geq 4.0$ & $0.05(0.01-0.42)$ & 0.005 \\
GGT (IU/L) & $<192$ & 1 & \\
& $\geq 192$ & $42.12(2.28-779.33)$ & 0.012 \\
WFA + -M2BP & $<2.0$ & 1 & 0.021 \\
\hline
\end{tabular}




\section{Figure Legends}

Figure 1. Correlation between serum WFA+-M2BP level and liver fibrosis score. Boxes represent the interquartile range of the data. The lines across the boxes indicate median values. $\mathrm{HC}$, healthy controls.

Figure 2. Receiver-operating characteristic curves of WFA+-M2BP, FIB-4 index, APRI, AST/ALT ratio, and Forn's index for the estimation of $(A)$ significant fibrosis $(\geq F 2),(B)$ severe fibrosis $(\geq F 3)$, and $(C)$ cirrhosis $(F 4)$.

Figure 3. Cumulative survival rates were analyzed using the Kaplan-Meier method according to baseline WFA+-M2BP levels. (A) Survival was significantly lower in patients when WFA ${ }^{+}-M 2 B P$ level was $\geq 2.0$. (B) Progression of liver decompensation was significantly higher in patients when WFA+-M2BP level was $\geq 2.0$. 